\title{
A Kinetic Approach to Photomineralization of Methane in Air by Membranes Based on $\mathrm{TiO}_{2} / \mathrm{WO}_{3}$
}

\author{
Ignazio Renato Bellobono ${ }^{1}$, Flavia Groppi ${ }^{2}$, Michela Sturini ${ }^{3}$, Angelo Albini ${ }^{3}$ and Franca Morazzoni ${ }^{4}$ \\ 1. LASA, Department of Physics, University of Milan, Segrate (MI) I-20090, Italy \\ 2. LASA, Department of Physics, University of Milan, and INFN (National Institute of Nuclear Physics), Segrate (MI) I-20090, Italy \\ 3. Department of Chemistry, University of Pavia, Pavia 27100, Italy \\ 4. Department of Materials Science, University of Milano Bicocca, Milan I-20126, Italy
}

\begin{abstract}
Photomineralization of methane in air (10.0-1,000 ppm (mass/volume) of C) at 100\% relative humidity (dioxygen as oxygen donor), was systematically studied at $318 \pm 3 \mathrm{~K}$, in an annular laboratory-scale reactor, by photocatalytic membranes immobilising titanium dioxide and tungsten trioxide as co-photocatalysts. Kinetics of both substrate disappearance, to yield intermediates, and total organic carbon (TOC) disappearance, to yield carbon dioxide, were followed. A kinetic model was employed, from which, by a set of differential equations, four final optimised parameters, $k_{1}$ and $K_{1}, k_{2}$ and $K_{2}$, were calculated, able to fit the whole kinetic profile adequately. Modelling of quantum yields, as a function of substrate concentration and irradiance, as well as of concentration of photocatalysts, was carried out very satisfactorily. Kinetics of hydroxyl radicals reacting between themselves, leading to hydrogen peroxide, other than with substrate or intermediates leading to mineralization, were considered, paralleled by second competition kinetics involving superoxide radical anion. When using appropriate blends of the two photocatalysts, limiting quantum yields $\Phi_{\infty}$ values increase considerably and approach the maximum allowable value for the investigated molecule, in a much wider range of irradiances than that shown by the single catalysts mainly at low irradiances. This may be interpreted by strong competition kinetics of superoxide radicals generated by the catalyst defects, in the corresponding range of high irradiances. By this way, operation at high irradiance values is possible, without losing any efficiency for the mineralization process.
\end{abstract}

Key words: Titanium dioxide and tungsten trioxide co-photo catalysts, photo catalytic membranes, quantum yields, gaseous methane mineralization, kinetic modelling.

\section{Introduction}

Photocatalytic processes on semiconductors are one of the newer AOP (Advanced Oxidation Processes) techniques. They were developed during around the last fifty years [1-9], starting from the early $70 \mathrm{~s}$, in the presence of catalysts, such as, among the most promising materials, titanium dioxide, with UV radiation, or tungsten trioxide in the visible range. If the UV radiation is from the sun, the treatment costs are reduced drastically, by transforming photocatalysis in one of the most appealing and environmentally friendly processes for complete mineralization of

Corresponding author: Ignazio Renato Bellobono, $\mathrm{PhD}$, CSci, CChem, FRSC, emeritus professor of chemistry, University of Milan, Community Foundation of Milan. organic pollutants. Furthermore, photocatalytic processes may be used as a pre-treatment of toxic chemicals, in order to convert them into fully biodegradable compounds to be finally processed by the most economical technology presently available, that of active sludge [10].

In all preceding papers on these topics (see, for example, Refs. [11-14]), the main concern has been devoted to the immobilization of the semiconductor photocatalyst and its photo-promoting agents in a membrane structure, in order to manufacture modular membrane reactors, the performance of which has been effectively estimated as one of the highest for energy efficiency [11].

From the point of view of mechanisms of surface photochemical reactions that take place in heterogeneous 
systems, a paper [15] has criticised the a priori assumed validity of the Langmuir-Hinshelwood kinetic model to interpret the experimental observations, as well as the assertion that the kinetics of a heterogeneous photoreaction are either first-order or half-order dependent on photon flow. Such a criticism had already been emphasized in the literature also from the point of view of engineering applications, and dates back to earlier pilot plant studies [16]. These have shown, for example, the influence of flow rate on apparent thermodynamic constant of adsorption/desorption, relative to the Langmuir-Hinshelwood model, as well as the inadequacy of this model to fit kinetics of mineralization, other than in the segment of the initial rate [12-14]. Particularly, when using immobilised photocatalysts, this pseudo-thermodynamic parameter of the Langmuir-Hinshelwood model should have a, partly at least, kinetic character. In aerated water, the photogenerated conduction band electrons reduce dissolved oxygen or other oxygen donors, added to this purpose, to yield $\mathrm{O}_{2}^{\bullet-}$, in acid-base equilibrium with $\mathrm{HO}_{2}{ }^{\bullet}$. The possibility of the latter reaction to take place quantitatively is of uttermost importance in environmental applications of photocatalyis, because, in the last case, oxidation of organic compounds may be carried out by $\mathrm{HO}_{2}^{\bullet}$ together with $\mathrm{OH}^{\bullet}$ radicals. This allows obtaining a fourfold maximum quantum yield with respect to that permissible if each absorbed photon would be able to produce a single hydroxyl radical only. This occurrence has been shown as effective [12, 16, 17] when using, for example, ozone as oxygen donor, or even dioxygen of air when promoting the activity of the immobilised semiconductor by suitable doping agents, such as $\mathrm{Co}(\mathrm{III})$ or $\mathrm{V}(\mathrm{V})$, coimmobilised with the semiconductor itself in a membrane structure, and acting as scavengers of generated photoelectrons.

Comparison of efficiency of different kinds of supported or immobilized photocalysts, and objective, quantitative characterization of their photocatalytic power are thus the major problems to define kinetic reactivity. In most of literature works, this kind of assessment is often made simply by matching qualitatively concentration profiles of degraded species. In addition, in many cases described in the literature, no regard is made to total organic carbon (TOC) mineralization, but merely to transformation of substrate into some intermediate compound, as the first step of the complicated reaction mechanism leading finally to carbon dioxide. Moreover, it is a common practice, even when quantitative evaluations are made, to consider the photocatalytic process as a first order reaction, while it has been definitely ascertained that apparent reaction order varies with concentration of substrate $[13,16]$ with an evident transition from a limiting zero order, at high concentrations, to a limiting first order at low concentrations. Modelling of photocatalytic reactions, on the contrary, in a wide range of concentrations, and over all the kinetic concentration profiles of TOC, leading to full mineralization, has been approached systematically in previous papers of this series [12-14, 18], mostly for processes carried out in aqueous solutions. This has been done also from the stand-point of quantum yields and energy efficiencies, a very important aspect, which is often neglected in the literature pertinent to photocatalysis.

In more recent papers, modelling has been extended to photocatalytic kinetic runs in gaseous phase, by using methane in air as a model molecule, both to apply the four parameters of kinetic model used successfully in preceding studies [18-20] for fitting the whole kinetic profile, and to broaden the application of this model, as a function of all variables which show an influence on the photocatalytic kinetics, and consequently play a primary role in all trustworthy characterization procedures of immobilised materials. The dependence of the four parameters of the kinetic model cited in Refs. [12-14, $18,20]$ on flow rate, on reactor geometry, on radiation flow, and on concentration of a proprietary photo promoter co-immobilized with the semiconductor in 
the photocatalytic membranes has been first examined. By this way, information, preliminarily investigated in a previous work [19] on methane in air, has been implemented and gets deeper. Secondly, the influence of these variables on the same parameters [12-14], relatively to experiments carried out in aqueous solutions has been checked, in order to systematically compare reactivity in liquid and gaseous phases. Finally, the dependence of quantum yields on concentration of substrate and on photon flow has been considered and analysed, with the goal of attaining, by all these variables, expressed in the most convenient ways, a complete rationalization of the photocatalytic process, both in liquid and gaseous phases, from the engineering point of view, as well from the perspective of establishing a reliable standardization method of the photocatalytic performance of materials.

In the present paper, the dependency of quantum yields of methane mineralization, in a gas phase, on composition of a photocatalytic blend formed by titanium dioxide and tungsten trioxide nanopowders, immobilized in membranes has been studied. This has mainly been done to compare marked increase of reactivity due to presence of photo promoters with behaviour of mixed photocatalysts. In fact, in recent years [21, 22], a growing interest has been concentrated in photocatalytic titanium dioxide and tungsten trioxide mixed oxides, in relation to electron transfer efficiency [21], particularly, to minimize electron-hole pairs recombination given by the use of titanium dioxide photo catalyst alone. Nevertheless, no reaction kinetic studies have been performed in recent literature until now that was able to suggest the reactions responsible for the enhancement of quantum yields.

\section{Materials and Methods}

\subsection{Reagents}

Methane was obtained from Fluka (GC purity greater than 99.8\%). It was used as received with no further purification. Ultra pure water, to be aerosolised in the reactor, when performing kinetic runs of methane in air, to maintain a $100 \%$ relative humidity at the operating temperature of the photoreactor (maximum contents of $\mathrm{Na}^{+}$and heavy metal ions 0.02 and $0.004 \mathrm{mg} \cdot \mathrm{kg}^{-1}$ respectively) was obtained by cross-flow ultrafiltration on composite membranes immobilizing active carbon and nuclear grade ion exchange resins, as described in Ref. [23]. Concentration of substrate in the gas phase was 10.0-1,000 ppm (mass expressed as carbon/volume).

\subsection{Photocatalytic Membranes}

The photocatalytic membranes (PHOTOPERM ${ }^{\mathbb{R}}$ BIT/333), kindly supplied by B.I.T. srl, Milan, I, were standard photocatalytic membranes, immobilizing 30 $\pm 3 \mathrm{wt} \%$ of a photocatalytic blend obtained by titanium dioxide (P25 by Degussa, Germany) and tungsten dioxide (50 $\mathrm{nm}$ nanopowder Pearl Yellow, by Wuhuhlangchen, China) as co-catalyst in the range $30-55 \mathrm{~mol} / \mathrm{mol} \%$ with respect to titanium. Photocatalytic membranes [16, 23-28], and more generally membranes, prepared by photografting, to immobilise reagents, catalysts, and sorbents [23-28], were described and characterized in previous studies. These membranes were prepared (patents pending) by grafting and graft polymerising, onto a non-woven microporous polypropylene tissue, $250 \pm 9 \mathrm{~g} \cdot \mathrm{m}^{-2}$ of a pre-polymeric blend containing $30 \pm 3 \mathrm{wt} \%$ of photocatalysts, under rheological control, in the presence of proprietary photoinitiating and photosensitising systems.

\subsection{Apparatus and Procedures}

The laboratory-scale photoreactor was substantially the same as that already described in Ref. [27].

Contrarily to procedure of a previous work [19], in which, when operating in the gaseous phase, an aqueous solution of hydrogen peroxide was aerosolized continuously into the reactor, in order to supply the stoichiometric amount of this oxygen donor, 
in the present work, only ultra pure water was aerosolized in the photoreactor, just to maintain $100 \%$ relative humidity at $318 \pm 3 \mathrm{~K}$, which was the operating temperature of the reactor. This was made, to check the influence on rate of the oxygen content of air, instead of using hydrogen peroxide. Overall volume of gaseous phase being treated was 4,000 \pm $0.005 \mathrm{~L}$, in standard experiments; the ratio between the overall reacting volume and the length of the lamp, corresponding to the length of the irradiated side of the membrane was $160 \pm 6 \mathrm{~cm}^{3} \cdot \mathrm{cm}^{-1}$. A high pressure mercury arc lamp with a nominal power of $1.00 \mathrm{~kW}$ was employed, kindly supplied by Chimia Prodotti e Processi (Muggiò, Milan, Italy): it was further provided with a step-by-step power regulation device, so that power absorbed by the membrane, as determined actinometrically, could be varied between 0.10 and $4.0 \mathrm{~W} \cdot \mathrm{cm}^{-1}$. The membrane being concentric to the lamp in the photoreactor used, absorbed power per unit length of lamp was also the same, if referred to unit length of membrane. At $0.30 \mathrm{~W} \cdot \mathrm{cm}^{-1}$ the overall power absorbed by the membrane, within the absorption range of immobilized semiconductor, when using titanium dioxide alone, in the experimental conditions of the present work, was $30 \mathrm{~W}$, that is 4.45 $\times 10^{-5}$ Einstein $\cdot \mathrm{s}^{-1}$. Owing to the refrigeration system of the lamp, by means of water circulating in the lamp sheath, made of quartz, mean temperature, during the runs, was $318 \pm 3 \mathrm{~K}$.

In the present, as well as in previous papers of this series, membranes were placed and fixed coaxially with the lamp, in such a way that the flow was perpendicular to the membrane. Owing to the microporous structure of the membrane, the pressure drop was very modest: it corresponded to 0.9-1.6 mm $\mathrm{Hg}$, when the flow rate of a liquid system was $4 \mathrm{~m}^{3} \cdot \mathrm{h}^{-1}$ in the laboratory-scale photoreactor.

The standard flow rate in gaseous phase kinetic runs, such as those examined in the present paper, to study the correlation between irradiance and composition in titanium dioxide - tungsten trioxide photocatalytic blend, was $300 \mathrm{~m}^{3} \cdot \mathrm{h}^{-1}$. In this latter experimental condition of flow rate, the ratio between the overall reaction volume and the length of the membrane was varied, substantially by varying the volume of reservoir, from and to the reservoir in which circulation of gaseous stream took place.

The disappearance of methane, as such, was followed by GC-MS quantitative analysis, as described in Ref. [20].

In order to get the values of quantum yields by the study of relationship between rate of methane disappearance, as such, and the overall rate of organic carbon disappearance, the latter was also followed, by total organic carbon (TOC) analysis. To this purpose, a Shimadzu TOC-V instrument was used, by which the contribution of inorganic carbon present was always evaluated, in order to obtain TOC by difference from total carbon and inorganic carbon determinations. Determinations were accurate within $20 \mathrm{ppb} \cdot \mathrm{C}$.

During all the duration of the runs, no sensible decrease of activity of the immobilized photocatalyst was observed, as checked both by random repetition of experiments at various initial concentrations of the hydrocarbon, and by reiteration of whole series of tests at the beginning and at the end of the prolonged period, throughout which experimentation has been carried out.

\subsection{Modelling of Quantum Yields}

Kinetic modelling was carried out by generalized procedures followed in preceding papers [20], in which details of kinetic model are illustrated. These procedures do not have the goal of proposing and checking photochemical mechanisms, but simply to reproduce closely experimental data, for engineering purposes, without any strict implication on the degradation mechanisms.

To sum up, if mineralization of substrate $\mathrm{S}$ to $\mathrm{CO}_{2}$ is supposed to occur through one single intermediate I, following Eq. (1): 


$$
\mathrm{S} \rightarrow \mathrm{I} \rightarrow \mathrm{CO}_{2}
$$

and if both $\mathrm{S}$ and $\mathrm{I}$ show a competitive apparent adsorption onto the immobilized semiconductor surface, as expressed by apparent adsorption constants $K_{1}$ and $K_{2}$ respectively, models for experimental data, used in non-linear regression analysis, may be expressed by the following system of first-order ordinary differential equations in the dependent variables $C_{\mathrm{S}}, C_{\mathrm{I}}$ and $C_{\mathrm{CO} 2}$ :

$$
\begin{gathered}
\left(\mathrm{d} C_{\mathrm{s}} / \mathrm{d} t\right)=-k_{1} \cdot K_{1} \cdot C_{\mathrm{s}} /\left(1+K_{1} \cdot C_{\mathrm{s}}+K_{2} C_{\mathrm{I}}\right) \\
\left(\mathrm{d} C_{\mathrm{I}} / \mathrm{d} t\right)=\left(k_{1} \cdot K_{1} \cdot C_{\mathrm{s}}-k_{2} \cdot K_{2} \cdot C_{\mathrm{I}}\right) / \\
\left(1+K_{1} \cdot C_{\mathrm{s}}+K_{2} \cdot C_{\mathrm{I}}\right) \\
\left(\mathrm{d} C_{\mathrm{CO} 2} / \mathrm{d} t\right)=k_{1} \cdot K_{1} C_{\mathrm{I}} /\left(1+K_{1} \cdot C_{\mathrm{s}}+K_{2} \cdot C_{\mathrm{I}}\right)
\end{gathered}
$$

where $C$ denotes concentrations of species to which the pertinent suffix refers, $t$ time; $k_{1}$ and $k_{2}$ the kinetic constants relative to degradation of $\mathrm{S}$ and $\mathrm{I}$ respectively. Intermediate I is supposed to simulate the behaviour of all the often uncountable, numerous intermediates, which are on the route from the substrate $\mathrm{S}$ to $\mathrm{CO}_{2}$. Experimentally speaking, only kinetic profiles of $\mathrm{S}$ and $\mathrm{CO}_{2}$ may be easily followed. Even if, for the molecule chosen in the present investigation, a limited number of intermediates may be reasonably envisaged from the substrate to $\mathrm{CO}_{2}$, the same is not true for aromatic and/or heterocyclic compounds, or still for long chain aliphatics, which have been the object of previous investigations.

Furthermore, the conservative balance of carbon has to be accounted and verified experimentally, all concentrations of substrate and intermediate being expressed in terms of carbon content, as obtained from TOC analysis:

$$
\begin{gathered}
C_{\mathrm{C}}(\text { at time } t)=C_{\mathrm{TOC}}(\text { at time } 0)=C_{\mathrm{S}}(\text { at time } t)+ \\
C_{\mathrm{I}}(\text { at time } t)+C_{\mathrm{CO} 2}(\text { at time } t)
\end{gathered}
$$

where $C_{\mathrm{C}}$ represents the total carbon, both organic and inorganic, carbon dioxide representing the final product of the mineralization process,

$$
C_{\mathrm{TOC}} \text { (at time } 0 \text { ) being equal to } C_{\mathrm{S}} \text { (at time } 0 \text { ) }
$$
and

$$
\begin{aligned}
& \left.\left.C_{\text {TOC }} \text { (at time } t\right) \text { being equal to } C_{\mathrm{S}} \text { (at time } t\right)+ \\
& \qquad C_{\mathrm{I}}(\text { at time } t)
\end{aligned}
$$

The major limitation of Eq. (6) is to regard only intermediates containing carbon, which is, anyway, the most common condition in degradation of organics. If this should not be the case, Eq. (6) should be substituted by Eq. (7):

$$
\begin{aligned}
& C_{\mathrm{C}}(\text { at time } t)=C_{\mathrm{TOC}}(\text { at time } 0)= \\
& C_{\mathrm{S}}(\text { at time } t)+C_{\mathrm{CO} 2}(\text { at time } t)
\end{aligned}
$$

and Eq. (4) by Eq. (8):

$$
\left(\mathrm{d} C_{\mathrm{CO} 2} / \mathrm{d} t\right)=-\left(\mathrm{d} C_{\mathrm{S}} / \mathrm{d} t\right)
$$

Eqs. (2)-(6) constitute a suitable model for temporal disappearance of TOC, which is a relevant analytical parameter to follow environmental pollution problems.

By using the optimised parameters of Eqs. (2)-(4) the rates of photomineralization were evaluated as a function of initial concentration, $C_{0}$, and consequently, by operating at a certain value of the photon flow, the corresponding quantum yields $\Phi_{0}$ were obtained, with reference to energy experimentally absorbed by the membrane.

Quantum yields $\Phi_{0}$, calculated from rates, followed an apparently Langmuirian function of initial concentration of substrate $C_{0}$, by which $\Phi_{\infty}$ values at "infinite" concentration could be obtained:

$$
1 / \Phi_{0}=1 / \Phi_{\infty}+1 /\left(\Phi_{\infty} C_{0} K\right)
$$

where the $K$ value substantially coincided with the lowest between the two $K_{1}$ and $K_{2}$ constants above, for many molecules being $K_{1}-K_{2}$. These values of "infinite" concentrations should be physically intended as "limiting" values, because they were experimentally approached and reached already at concentrations $C_{0}$ of about $1 \times 10^{-3} \mathrm{M}$.

Finally, by repeating all sets of experiments, at different values of the absorbed radiant power per unit length of photocatalytic membrane, which was varied in the range $0.10-4.0 \mathrm{~W} \cdot \mathrm{cm}^{-1}$, and by applying Eq. (14) to each set of these experiments, the $\Phi_{\infty}$ values as a function of the absorbed radiant power, per unit length of photocatalytic membrane, were obtained.

In the present paper, in order to obtain quantum yields as a function of composition of the 
photocatalytic blend, the procedure described in the preceding paragraph was adopted for each composition of the blend, and the $\Phi_{\infty}$ values obtained at each value of composition examined were potted as a function of absorbed power per unit membrane length.

\section{Results and Discussion}

Photomineralization of methane in air at $100 \%$ relative humidity, and in a concentration range corresponding to $10.0-1,000 \mathrm{ppm}$ (mass/volume) of carbon, was studied at $318 \pm 3 \mathrm{~K}$, in a laboratory-scale reactor, by using dioxygen of air as oxygen donor, and by photocatalytic membranes immobilising $30 \pm 3 \%$ of a photocatalytic blend constituted by titanium dioxide and tungsten dioxide as a function of absorbed power per unit length of membrane, which was expressed in $\mathrm{W} \cdot \mathrm{cm}^{-1}$. The latter was varied in the range $0.15-4.0 \mathrm{~W} \cdot \mathrm{cm}^{-1}$. This is a convenient measure of power absorbed per unit apparent geometrical membrane surface, since, owing to the annular geometry of photoreactor, absorbed power per unit length, divided by membrane circumference, yields exactly that value. Kinetics of both substrate disappearance, to yield intermediates, and total organic carbon (TOC) disappearance, to yield carbon dioxide, were followed. The four parameters obtained for photo catalytic degradation of methane by use of titanium dioxide alone as catalyst, at various values of absorbed power per unit length of membrane, were reported in Table 1.

By Eq. (9), in which the $K$ values substantially coincided with $K_{2}$ of the four parameters kinetic model, $k_{1}$ and $K_{1}, k_{2}$ and $K_{2}$ (see Section 2) and by applying the procedure described above (see Section 2 ), the $\Phi_{\infty}$ values as a function of the absorbed radiant power, per unit length of photocatalytic membrane, expressed in $\mathrm{W} \cdot \mathrm{cm}^{-1}$ were obtained, and reported in Fig. 1.

To explain the behaviour of sigmoid curves, such as those of Fig. 1, both for experiments carried out with only titanium dioxide, in a previous work [20], or with tungsten trioxide alone as photocatalysts, in the present work, competition kinetics of the reaction of hydroxyl radicals with themselves to give hydrogen peroxide, accompanied by the reaction of these radicals, as well as of superoxide anion radical and/or its conjugate acid, with the substrate and intermediates, leading to mineralization may be envisaged, such as given by reactions $(a)$ on one side, and reactions $(b)$ and $\left(b^{\prime}\right)$ on the other.

Reaction (a) corresponds to the formation of hydrogen peroxide by recombination of hydroxyl radicals, $\mathrm{d} C_{\mathrm{H} 2 \mathrm{O} 2} / \mathrm{d} t$, where $t$ is time:

$$
\cdot \mathrm{OH}+{ }^{\bullet} \mathrm{OH} \rightarrow \mathrm{H}_{2} \mathrm{O}_{2}
$$

Table 1 Parameters, $k_{1}$ and $K_{1}, k_{2}$ and $K_{2}$, able to fit the whole photomineralisation curve, following Eq. (1), at various values of absorbed power per unit length of membrane expressed in $\mathrm{W} \cdot \mathrm{cm}^{-1}$. They were obtained by optimisation of kinetic curves, by using the set of differential equations corresponding to kinetic model of Eq. (1). All the parameters are expressed in ppm of carbon (mass of $\mathrm{C} / \mathrm{volume}, \mathrm{mg} \cdot \mathrm{L}^{-1}$ ) and refer to the photodegradation of methane in gaseous phase by dioxygen of air as oxygen donor (treated volume of the gas phase $4.00 \mathrm{~L}$; membrane length $100.0 \pm 0.5 \mathrm{~cm}$; ratio between overall reaction volume and membrane length $160 \pm 6 \mathrm{~cm}^{3} \cdot \mathrm{cm}^{-2}$; membranes immobilizing $30 \pm 3 \mathrm{wt} \% \mathrm{TiO}_{2}$.

\begin{tabular}{lllll}
\hline $\begin{array}{l}\text { Absorbed power per } \\
\text { unit membrane length } \\
\left(\mathrm{W} \cdot \mathrm{cm}^{-1}\right)\end{array}$ & $\begin{array}{l}k_{1} \\
\left(\mathrm{ppm} \cdot \mathrm{C} \cdot \mathrm{min}^{-1}\right)\end{array}$ & $\begin{array}{l}k_{2} \\
\left(\mathrm{ppm} \cdot \mathrm{C} \cdot \mathrm{min}^{-1}\right)\end{array}$ & $\begin{array}{l}K_{1} \\
(\mathrm{ppm} \cdot \mathrm{C})^{-1}\end{array}$ & $\begin{array}{l}K_{2} \\
(\mathrm{ppm} \cdot \mathrm{C})^{-1}\end{array}$ \\
\hline 0.15 & $0.125(8)$ & $0.290(8)$ & $0.0129(8)$ & $0.0135(9)$ \\
0.30 & $0.242(6)$ & $0.592(8)$ & $0.0134(9)$ & $0.0114(8)$ \\
0.90 & $0.596(3)$ & $1.49(3)$ & $0.0138(5)$ & $0.0129(6)$ \\
1.50 & $0.815(4)$ & $1.86(4)$ & $0.0124(4)$ & $0.0099(6)$ \\
2.00 & $0.873(3)$ & $2.44(3)$ & $0.0131(5)$ & $0.0100(7)$ \\
3.00 & $0.895(4)$ & $2.38(5)$ & $0.0129(5)$ & $0.0108(7)$ \\
4.00 & $0.890(5)$ & $2.37(6)$ & $0.0131(6)$ & $0.0136(9)$ \\
\hline
\end{tabular}

Uncertainties are indicated between parentheses and expressed, relatively to the last digits, as probable errors of the means of optimised values from nine sets of runs in the range of concentrations tested. 


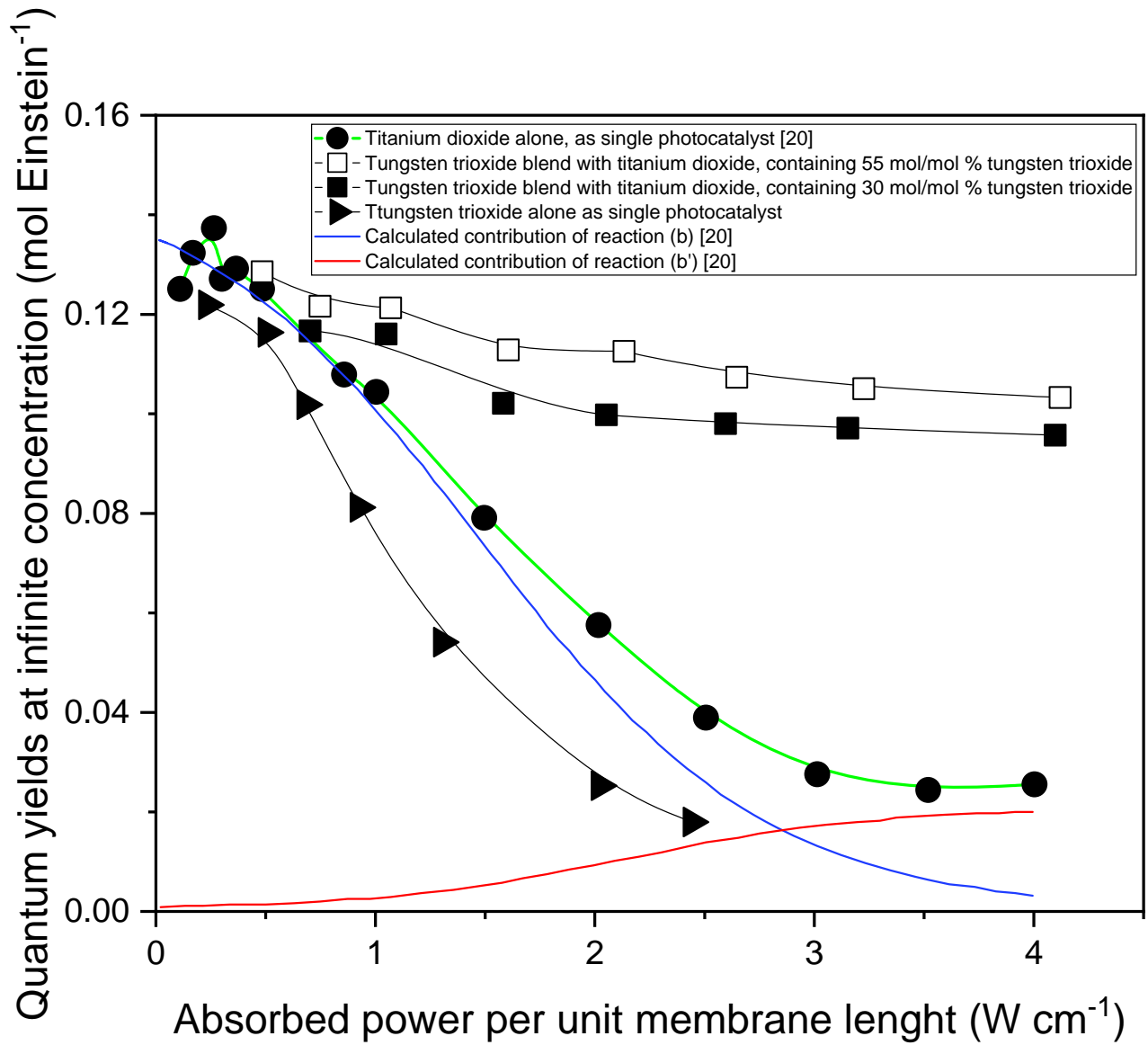

Fig. 1 Quantum efficiencies $\Phi_{\infty}\left(\mathrm{mol} \cdot\right.$ Einstein $\left.^{-1}\right)$ of methane, extrapolated by Eq. (1), at "infinite" concentration of substrate, relative to measurements in the gaseous phase, as a function of absorbed power per unit length of irradiated photocatalytic membrane, expressed as $\mathrm{W} \cdot \mathrm{cm}^{-1}$, evaluated by photocatalytic membranes immobilising:

(1) titanium dioxide alone [20] as single photocatalyst (black dots);

(2) tungsten trioxide alone as single photocatalyst (black triangles);

(3) tungsten trioxide blend with titanium dioxide, containing $30 \mathrm{~mol} / \mathrm{mol} \%$ tungsten trioxide (black squares);

(4) tungsten trioxide blend with titanium dioxide, containing $55 \mathrm{~mol} / \mathrm{mol} \%$ tungsten trioxide (empty squares).

The blue curve represents the calculated contribution of reaction $(b)$, as modelled in Ref. [20], decreasing with increasing power.

The red curve represents the calculated contribution of reaction $(b$ '), as modelled in Ref. [20], increasing with increasing power.

The green represents equation modelled in Ref. [20], as a sum of both $(b)$ and $\left(b^{\prime}\right)$ contributions.

and its rate $r_{a}$ may be written as:

$$
r_{a}=k_{a} \cdot C_{\bullet \mathrm{OH}^{2}}
$$

where $k_{a}$ is the rate constant of reaction $(a)$, and $C_{\bullet \mathrm{OH}}$ the concentration of hydroxyl radicals.

Reaction $(b)$ is given by:

$\bullet \mathrm{OH}+$ substrate (or intermediates) $\rightarrow$ mineralization

and its rate $r_{b}$ may be written as:

$$
r_{b}=k_{b} \cdot C_{\mathrm{S}} \cdot C_{\bullet \mathrm{OH}}
$$

where $k_{b}$ is the rate constant of reaction $(b)$, and $C_{\mathrm{S}}$ is the concentration of substrate or intermediates, on the surface of the photocatalytic membrane on to which mineralization takes place.

Reaction $\left(b^{\prime}\right)$ by the $\mathrm{O}_{2}^{\bullet-} / \mathrm{HO}_{2}{ }^{\bullet}$ superoxide radical couple is given by:

$$
\begin{gathered}
\mathrm{O}_{2}{ }^{\bullet-} / \mathrm{HO}_{2}{ }^{\bullet}+\text { substrate (or intermediates) } \rightarrow \\
\text { mineralization }
\end{gathered}
$$

and its rate $r_{b}$, may be written as:

$$
r_{b},=k_{b} \cdot C_{\mathrm{S}} \cdot C_{\mathrm{O} 2 \bullet-/ \mathrm{HO} 2 \bullet}
$$


where $k_{b}$, is the rate constant of reaction $\left(b^{\prime}\right)$.

At very low photon flows, corresponding to the upper plateau of curves 1 of Fig. 1, at which the concentration of hydroxyl radicals formed by irradiation onto the semiconductor surface is relatively low, reaction $(a)$ is certainly negligible, with respect to reaction $(b)$. Furthermore, in these circumstances, in the presence of oxygen donor, scavenging of photogenerated electrons of the conduction band $\mathrm{e}_{\mathrm{CB}}^{-}$ should be quantitative and compatible with reactions (c) and (d) occurring at comparable rates, and followed by reaction $(e)$.

$$
\begin{gathered}
\mathrm{e}_{\mathrm{CB}}^{-}+\mathrm{H}_{2} \mathrm{O}_{2} \rightarrow{ }^{\bullet} \mathrm{OH}+\mathrm{OH}^{-} \\
\mathrm{e}_{\mathrm{CB}}^{-}+\mathrm{O}_{2} \rightarrow \mathrm{O}_{2}^{\bullet-} / \mathrm{HO}_{2}^{\bullet} \\
\mathrm{HO}_{2}^{\bullet}+{ }^{\bullet} \mathrm{OH} \rightarrow \mathrm{O}_{2}+\mathrm{H}_{2} \mathrm{O}
\end{gathered}
$$

If these conditions are working, a quantum efficiency equal or very near to the maximum one allowable may be attained. This hypothesis obviously corresponds to stating that each absorbed photon, in the optimal conditions of a membrane photoreactor, should give rise to a hydroxyl radical, at every $C_{0}$ value of Eq. (9). Following this same hypothesis, reactivity of conduction band electrons, in the presence of hydrogen peroxide as oxygen donor, should give rise, almost quantitatively, to molecular oxygen, or, alternatively to reactions $(c)-(e)$, to reduced species, in the semiconductor lattice or at the interface, by reaction with donors (water itself included). If these reactions take place quantitatively, also the contribution of $r_{b}$, to the overall rate $r$ given by:

$$
r=r_{a}+r_{b}+r_{b}
$$

which will be negligible. Radical reactivity leading to integral mineralization of substrate should be controlled by Eq. (13), with both $r_{a}$ and $r_{b}$, much lower than $r_{b}$ and consequently the $r_{b} / r$ factor should reach the maximum value permissible (approaching the unity). This situation is surely achieved at very low values of the photon flow, since effectively, in these conditions, in Fig. 1 the maximum allowable quantum yields for methane mineralization are operative, corresponding to reactivity of hydroxyl radicals alone, as shown by the apparent upper plateau of this curve. These values are very near to 0.125 mol-Einstein ${ }^{-1}$, which corresponds to mineralization of methane by 8 hydroxyl radicals, as required by stoichiometry. Typically, as stated above (see Section 2 ), at $0.30 \mathrm{~W} \cdot \mathrm{cm}^{-1}$ (a representative value in the range of $0-0.5 \mathrm{~W} \cdot \mathrm{cm}^{-1}$, where maximum quantum yields of Fig. 1 are operative, and consequently the $r_{b} / r$ ratio is unitary) this photon flow amounted to $4.45 \times 10^{-5}$ Einstein $\cdot \mathrm{s}^{-1}$. This means that, for each $\mathrm{cm}$ of membrane length, and consequently for each $\mathrm{cm}$ of length of the photoreactor, photogeneration of $4.45 \times$ $10^{-5}$ moles (of hydroxyl radicals) $\mathrm{s}^{-1} \cdot \mathrm{cm}^{-1}$ took place. To transform this value in amount of hydroxyl radicals generated per unit time and per unit reactor volume, one should consider that in the laboratory scale module, given its overall volume in standard conditions, and owing to engineering design, the volume corresponding to each $\mathrm{cm}$ of membrane length was $160 \pm 6 \mathrm{~cm}^{3} \cdot \mathrm{cm}^{-1}$ (see Section 2). As a consequence, the production rate of ${ }^{\bullet} \mathrm{OH}$ radicals, always at the given absorbed power per unit length of $0.30 \mathrm{~W} \cdot \mathrm{cm}^{-1}$, was calculated as $1.11 \times 10^{-3} \mathrm{~mol} \cdot \mathrm{s}^{-1} \cdot \mathrm{L}^{-1}$. This linear relationship allows evaluating the production rate of hydroxyl radicals in the upper apparent plateau of curves such as that of Fig. 1, at each value of $\mathrm{W} \cdot \mathrm{cm}^{-1}$.

This mechanism, however could not rule also at high photon flows, when the quantum yields of Fig. 1, from the upper apparent plateau begin to decrease with increasing photon flow, and finally reach an apparent lower second plateau. This may be due, on one side, to the fact that the production rate of hydroxyl radicals, at $\mathrm{W} \cdot \mathrm{cm}^{-1}$ values higher than about 0.5 , should vary with photon flow by a non-linear relationship, and their evaluation is possible, by supposing that $C_{\bullet} \mathrm{OH}$ decreases exponentially with increasing $\mathrm{W} \cdot \mathrm{cm}^{-1}$ values, as:

$$
C \cdot \mathrm{OH}=f \times \mathrm{e}^{-g(\mathrm{~W} / \mathrm{cm})}
$$

with $f$ and $g$ positive constants. 
On the other side, with increasing photon flow, and with decreasing rate of hydroxyl radicals production, the rate of generation of superoxide radical couples, $\mathrm{O}_{2}{ }^{\bullet-} / \mathrm{HO}_{2}{ }^{\bullet}$, increases.

The concentration of these superoxide radical couples, as a function of absorbed power per unit length of membrane, may be assumed to vary according to an equation of the same kind as Eq. (14), but with a negative $g$ constant, as the production of these radicals should increase with increasing irradiance, in a complementary way by which hydroxyl radicals decrease.

Reaction ( $\left.b^{\prime}\right)$ thus yields a supplementary contribution to mineralization, besides that due to the photocatalytic reactivity of holes onto the semiconductor surface. In other words, to the $r_{b}$ contribution to mineralization, a further contribution $r_{b}$ should be added, deriving from the concentration of superoxide radical anion and/or its conjugate acid generated by reaction $(d)$. The overall contribution to mineralization, $\left(r_{b}+r_{b}\right) / r$, can be approximated by a weighted sum of the two contributions $(b)$ and $\left(b^{\prime}\right)$ :

$$
\begin{gathered}
\left(r_{b}+r_{b},\right) / r \cong y_{(\text {calc })}=\mathrm{f}_{1} /\left[1+\mathrm{e}^{-g}\left(\mathrm{~W}-\mathrm{W}_{1}\right) / \mathrm{cm}_{1}\right]+ \\
\mathrm{f}_{2} /\left[1+\mathrm{e}^{-g}\left(\mathrm{~W}_{2}\left(\mathrm{~W}_{2}\right) / \mathrm{cm}\right]\right.
\end{gathered}
$$

The error sum, $\mathrm{G}\left(\mathrm{f}_{1}, g_{1}, W_{1}, \mathrm{f}_{2}, g_{2}, W_{2}\right)=\sum_{\mathrm{i}=1}{ }^{n} w_{i}$ $\left[\mathrm{y}_{(\text {exp })}^{i}-\mathrm{y}_{(\text {(calc) }}^{i}\right]^{2}$, is minimized with respect to $\mathrm{f}_{1}, g_{1}$, $W_{1}, \mathrm{f}_{2}, g_{2}$ and $W_{2}$ parameters, to find the best model [20] that fits the data. As shown in Fig. 1 for methane when using only titanium dioxide as photocatalyst, the calculated curve (red curve) perfectly fits experimental data, and gives rise to a sigmoid curve, with two apparent plateaux values, as found experimentally. In Fig. 1, the contributions to quantum yields given by these two processes, for experiments carried out with titanium dioxide alone [20] have been plotted as a function of $\mathrm{W} \cdot \mathrm{cm}^{-1}$. It clearly appears that the contribution (blue curve) of hydroxyl radicals to mineralization decreases with irradiance, while the contribution (mauve curve) of superoxide anion radical and its conjugate acid increases. To sum up, only the model given by both reactions $(b)$ and $\left(b^{\prime}\right)$, in competition with $(a)$, is completely compatible with experimental data, and able to represent, fully satisfactorily, the dependence of quantum yields on irradiance values. In other words, with photocatalytic membranes based on the pure photocatalysts examined in the present study, the radical reaction model above allows reaction $\left(b^{\prime}\right)$ to occur only at very high irradiance values, even higher than $4 \mathrm{~W} \cdot \mathrm{cm}^{-1}$.

For the experiments carried out by photocatalytic membranes co-immobilizing tungsten trioxide together with titanium dioxide, the sigmoid shape of curves of Fig. 1 changed with increasing concentration of titanium dioxide as co-photocatalyst, up to about a concentration of $45 \mathrm{~mol} / \mathrm{mol} \%$ of this latter (with respect to the sigmoid curve relative to tungsten trioxide alone) and the curves were flattened, as reported in the example of Fig. 1. It may effectively be seen that, when using a co-catalysts blend of this composition, the $\Phi_{\infty}$ values remain very near to the maximum allowable one $\left(0.125 \mathrm{~mol} \cdot\right.$ Einstein $\left.^{-1}\right)$, and very moderately decrease only when absorbed power per unit membrane length exceeds about $1 \mathrm{~W} \cdot \mathrm{cm}^{-1}$. This behaviour apparently mimics that of photo promoters studied in previous paper [20]. In these conditions, one may guess that reaction $(b)$ is the most prevalent mechanism, both at low and high irradiances, giving the maximum allowable values of quantum yields at substantially any value of irradiance. On the kinetic side, this may be interpreted by strong competition kinetics of superoxide radicals in the range of high irradiances due to charge separation of catalyst, and consequent electron transfer from conduction band of titanium dioxide to that of tungsten trioxide, followed by migration to dioxygen without recombination. On the chemical engineering side, this allows high efficiencies, and therefore the best design for photocatalytic membrane reactors, even when operating at high irradiance values, and consequently at high rates, without losing any efficiency for the mineralization process. 
On the contrary, this kinetic mechanistic modelling, which has found so many experimental confirmations in photocatalytic membrane reactors, both in liquid as well as in gaseous phase mineralisations of a variety of molecules [13, 14, 20, 29, 30], seems to be ruled out for mineralisation of formic acid, which is anyway the last mineralization step leading from methane to carbon dioxide, when titanium dioxide-tungsten trioxide co-photocatalysts are employed [21]. This, however, does not speak necessarily against modeling of the present and previous papers. Many supporting reasons may be allowed for. First of all, our modeling, aiming to engineering purposes, does not take into consideration neither single step of the mineralization mechanism, nor step-by-step mechanisms, but mediates the behavior of usually very large numbers of steps. Secondly, a membrane process is used, and immobilization of photocatalysts and/or doping agents, in a reliable membrane structure, occurs by a photo-grafting technology, completely different from those used in most studies for preparation of photocatalysts, and surely equivalently, or even much more efficient [11]. Furthermore, and perhaps more importantly, photocatalytic membranes, described in the present and previous papers are prepared by photografting processes, which imply the use and therefore the presence of $\mathrm{Co}(\mathrm{III})$ as well as of $\mathrm{V}(\mathrm{V})$ photo-sensitizers and photopromoters, able to support facilitated oxygen transport [25] as has been shown, and consequently to drive the peculiar mechanisms outlined above. Finally, titanium dioxide nano-powder simply dispersed in polymeric membranes, in the absence of any photo-promoter, was tested as photocatalyst in the phenol mineralization reaction assisted by molecular oxygen [31]. Kinetics experiments revealed that, although embedded, the oxide maintains significant catalytic activity. This result was first attributed to the homogeneous dispersion inside the polymeric host of titanium dioxide nanocrystals, which can be easily irradiated and interact with the reactants. Further investigation of the photogenerated charge carriers in the photocatalyst demonstrated that however electrons are trapped on $\mathrm{Ti}^{3+}$ defective centers, while holes are trapped on $\mathrm{C}$-centered species of the polymer matrix. In the presence of $\mathrm{O}_{2}$, the $\mathrm{C}$-centered radicals of the polymer transform into peroxy radicals, reinforcing the charge separation in the polymer-embedded oxide with respect to the powder. The positive interference of the polymer matrix in reinforcing the electron-hole separation is thus responsible for the relevant photoactivity, besides any action due to the photo grafting procedure and to the presence of photo-sensitizers and/or photo-promoters during the industrial preparation of photo catalytic membranes.

\section{Conclusions}

Quantum yields of mineralization of substrates in annular photoreactors immobilising the photocatalyst in a membrane structure show a Langmuirian-type dependency on initial concentration of substrates themselves, by which limiting quantum yields, corresponding to "infinite" concentration may be calculated, together with an apparent thermodynamic constant, coinciding, in a four parameters model (see Section 2 as well as Section 3), with the lowest between $K_{1}$ and $K_{2}$ values, very often being $K_{1}-K_{2}$.

These limiting quantum yields depend on radiant power absorbed per unit membrane length, in the cylindrical geometry of photoreactors.

Two kinds of behaviours are apparent, relatively to the use of single photocatalysts or of co-catalysts:

(1) When a single photocatalyst is employed, in the low radiant power range limiting quantum yields take the shape of a plateau corresponding to the maximum allowable quantum yields, evaluated on the basis of a single hydroxyl radical produced per each absorbed photon, thus showing the excellent performance of the photocatalytic membrane reactors. On the contrary, at high radiant power values, another apparent plateau is evident, at a value of about $1 / 5$ (for the experimental case of methane) with respect to the maximum value. 
This was interpreted on the basis of a competition kinetics of hydroxyl radicals with themselves, leading to hydrogen peroxide formation, other than with substrate or intermediates molecules leading to mineralization. In this model the contribution of hydroxyl radicals to mineralization strongly decreases with irradiance, while the contribution of superoxide anion radical and its conjugate acid increases. Two radical reactions thus appear to be responsible for mineralization, that of ${ }^{\circ} \mathrm{OH}$ radicals, and that of the $\mathrm{O}_{2}{ }^{\bullet-} / \mathrm{HO}_{2}{ }^{\bullet}$ radical couple, the last of which acquires importance only in the experimental conditions of high radiating power. If these contributions are considered together, in a weighted form, the calculated equation perfectly fits experimental data, and gives rise to a sigmoid curve, with two apparent plateaux values, as found experimentally. Noteworthy, it appears that the calculated curve of Fig. 1, as a sum of the two mainly contributing reactions given by hydroxyl and superoxide radicals, very satisfactorily coincides with experimental data, thus giving strikingly outstanding evidence to the righteousness of our model.

(2) When an appropriate co-photocatalytic system is employed, such as that given by tungsten trioxide and titanium dioxide photocatalysts, its action may be easily quantified and rationalised by measuring quantum yields at infinite concentration, as a function of irradiance and as a function of concentration of the co-photocatalysts co-immobilized in the photocatalytic membrane. When increasing concentration of the active photocatalyst, with respect to the other, above an apparent threshold value, for which no effect could be observed, $\Phi_{\infty}$ values increase considerably and approach the maximum allowable for the investigated molecule, in a much wider range of irradiances than that shown by the single catalysts. This should mean that the decrease of quantum yield brought about by the increase of irradiance, for single photocatalyst, and interpreted by a recombination mechanism of hydroxyl radicals, may be fully compensated by more important contributions of superoxide radicals.

\section{Acknowledgements}

The present paper is the 91st in a series of papers, authored by Ignazio Renato Bellobono and his collaborators over the past 35 years, which is collectively referred to as the "Photosynthetic Membranes" series.

\section{References}

[1] Linsebigler, A. L., Lu, G., and Yates Jr., J. T. 1995. "Photocatalysis on $\mathrm{TiO}_{2}$ Surfaces: Principles, Mechanisms, and Selected Results." Chem. Rev. 95: 735-58. https://doi.org/10.1021/cr00035a013.

[2] Hoffmann, M. R., Martin, S. T., Choi, W., and Bahnemann, D. W. 1995. "Environmental Applications of Semiconductor Photocatalysis." Chem. Rev. 95: 69-96. https://doi.org/10.1021/cr00033a004.

[3] Legrini, O., Oliveros, E., and Braun, A. M. 1993. "Photochemical Processes for Water Treatment." Chem. Rev. 93: 671-98. https://doi.org/10.1021/cr00018a003.

[4] Zheng, H., Ou, J. Z., Strano, M. S., Kaner, R. B., Mitchell, A., and Kalantar-Zadeh, K. 2011. "Nanostructured Tungsten Oxide-Properties, Synthesis, and Applications." Adv. Funct. Mater. 21: 2175-96. https://doi-org.pros.lib.unimi.it:2050/10.1002/adfm.2010 02477.

[5] Xu, H., Ouyang, S., Liu, L., Reunchan, P., Umezava, N., and Ye, J. 2014. "Recent Advances in $\mathrm{TiO}_{2}$-Based Photocatalysis." J. Mater. Chem. A 2: 12642-61. https://doi.org/10.1039/c4ta00941j.

[6] Mills, A., Davies, R. H., and Worsley, D. 1993. "Water Purification by Semiconductor Photocatalysis." Chem. Soc. Rev. 6: 417-25. https://doi.org/10.1039/CS9932200417.

[7] Prairie, M. R., Stange, B. M., Evans, L. R., and Martinez, S. L. 1993. "An Investigation of $\mathrm{TiO}_{2}$ Photocatalysis for the Treatment of Water Contaminated with Metals and Organic Chemicals.” Environ. Sci. Technol. 27: 1776-82. https://doi.org/10.1021/es00046a003.

[8] Schiavello, M. 1987. Photocatalysis and Environment: Trends and Applications. NATO ASI Series C. London, UK: Kluwer.

[9] Bahnemann, D. 1999. "Photocatalytic Detoxification of Polluted Waters." In The Handbook of Environmental Chemistry, edited by Boule, P. Berlin: Springer, 185-212.

[10] Bellobono, I. R., Barni, B., and Gianturco, F. 1995. "Induction of Integral Biodegradability in 
Non-biodegradable Waters by Advanced Photooxidation on Membranes Immobilizing Titanium Dioxide and Promoting Photocatalysts." J. Membrane Sci. 102: 139-47. https://doi.org/10.1016/0376-7388(94)00273-2.

[11] Dijkstra, M. F. J., Koerts, E. C. B., Beenackers, A. A. C. M., and Wesselingh, J. A. 2003. "Performance of Immobilized Photocatalytic Reactors in Continuous Mode." Am. Inst. Chem. Eng. J. 49: 734-44. https://doi.org/10.1002/aic.690490317.

[12] Ascari, F., Bellobono, I. R., and Tozzi, P. M. 2003. "Kinetic Modelling of Pilot Plant Photomineralization of Aqueous Solutions of Cibacron CR, as Model Molecule of Industrial Azo Dyes, on to Membranes Immobilizing Titanium Dioxide." Fresenius Environ. Bull. 12: 1195-201.

[13] Bellobono, I. R., Ascari, F., Lagrasta, C., Pinacci, P. L., Tozzi, P. M., Di Carlo, M. S., and Simoncelli, C. 2003. "Kinetic Modelling of Photomineralization of Phenol, as Model Molecule of Aromatic Micropollutants, and Validation of a Photochemical Reactor, Based on Photocatalytic Membranes Immobilizing Titanium Dioxide and Promoting Photocatalysts." Fresenius Environ. Bull. 12: 1536-44.

[14] Bellobono, I. R., Morazzoni, F., Bianchi, R., Mangone, E. S., Stanescu, R., Costache, C., and Tozzi, P. M. 2005. "Laboratory-Scale Photomineralisation of $n$-Alkanes in Aqueous Solution, by Photocatalytic Membranes Immobilising Titanium Dioxide." Int. J. Photoen. 7: ID718501. https://doi.org/10.1155/S1110662X05000127.

[15] Emeline, A. V., Ryabchuk, V. K., and Serpone, N. 2005. "Dogmas and Misconceptions in Heterogeneous Photocatalysis. Some Enlightened Reflections." J. Phys. Chem. 109: 18515-21. https://doi.org/10.1021/jp0523367.

[16] Barni, B., Cavicchioli, A., Riva, E., Zanoni, L., Bignoli, F., Bellobono, I. R., Gianturco, F., De Giorgi, A., Muntau, H., Montanarella, L., Facchetti, S., and Castellano, L. 1995. "Pilot-Plant-Scale Photodegradation of Phenol in Aqueous Solution by Photocatalytic Membranes Immobilizing Titanium Dioxide (PHOTOPERM ${ }^{\circledR}$ Process)." Chemosphere 30: 1861-74. https://doi.org/10.1016/0045-6535(95)00067-I.

[17] Gianturco, F., Chiodaroli, C. M., Bellobono, I. R., Raimondi, M. L., Moroni, A., and Gawlik, B. 1995. "Pilot-Plant Photomineralization of Atrazine in Aqueous Solution, by Photocatalytic Membranes Immobilizing Titanium Dioxide and Promoting Photocatalysts." Fresenius Environ. Bull. 6: 461-8.

[18] Bellobono, I. R., De Martini, G., Tozzi, P. M., Canevali, C., Morazzoni, F., Scotti, R., and Bianchi, R. 2006. "Modelling of Quantum Yields in Photocatalytic Membrane Reactors Immobilising Titanium Dioxide." Int. J. Photoen. https://doi.org/10.1155/IJP/2006/26870.
[19] Bellobono, I. R., Stanescu, R., Costache, C., Canevali, C., Morazzoni, F., Scotti, R., Bianchi, R., Mangone, E. S., De Martini, G., and Tozzi, P. M. 2006. "Laboratory-Scale Photomineralisation of $n$-Alkanes in Gaseous Phase, by Photocatalytic Membranes Immobilising Titanium Dioxdide." Int. J. Photoen. https://doi.org/10.1155/IJP/2006/73167.

[20] Bellobono, I. R., Rossi, M., Testino, A., Morazzoni, F., Bianchi, R., De Martini, G., Tozzi, P. M., Stanescu, R., Costache, C., Bobirica, L., Bonardi, M. L., and Groppi, F. 2008. "Influence of Irradiance, Flow Rate, Reactor Geometry, and Photopromoter Concentration on Mineralization Kinetics of Methane in Air and in Aqueous Solutions by Photocatalytic Membranes Immobilizing Titanium Dioxide." Int. J. Photoen. ID 28374. https://doi.org/10.1155/2008/283741.

[21] Dozzi, M. V., Marzorati, S., Longhi, M., Coduri, M., Artiglia, L., and Selli, E. 2016. "Photocatalytic Activity of $\mathrm{TiO}_{2}-\mathrm{WO}_{3}$ Mixed Oxides in Relation to Electron Transfer Efficiency." Appl. Catal. B-Environ. 186: 157-65. https://doi.org/10.1016/j.apcatb.2016.01.004.

[22] Dong, F., Dong, P., Hou, G., Xi, X., and Shao, R. 2017. "WO -Based Photocatalysts: Morphology Control, Activity Enhancement and Multifunctional Applications." Environ. Sci-Nano 4: 539-57. https://doi.org/10.1039/C6EN00478D.

[23] Bellobono, I. R., Tacchi, R., Selli, E., and Muffato, F. 1989. "Photosynthetic Membranes for Pure and Ultra Pure Water Production.” In Membrane Separation Processes, edited by Green, A. Bedford, UK: BHRA, 187-93.

[24] Barni, B., Cavicchioli, A., Riva, E., Zanoni, L., Bignoli, F., Bellobono, I. R., Gianturco, F., De Giorgi, A., Muntau, H., Montanarella, L., Facchetti, S., and Castellano, L. 1995. "Laboratory-Scale Photodegradation of Phenol in Aqueous Solution by Photocatalytic Membranes Immobilizing Titanium Dioxide." Chemosphere 30: 1847-60. https://doi.org/10.1016/0045-6535(95)00066-H.

[25] Bellobono, I. R., Muffato, F., Ermondi, C., Selli, E., and Righetto, L. 1991. "Gas Separation Membranes Containing Carbon Immobilized by Photochemical Grafting onto Polymers." J. Membrane Sci. 55: 273-81. https://doi.org/10.1016/S0376-7388(00)80582-7.

[26] Bellobono, I. R., Muffato, F., Ermondi, C., Selli, E., Righetto, L., and Zeni, M. 1991. "Thermodynamic Study of Sorption of $n$-Alkanes onto 5A Zeolites Immobilized in Photosynthetic Membranes." J. Membrane Sci. 55: 263-72. https://doi.org/10.1016/S0376-7388(00)80581-5.

[27] Bellobono, I. R., Selli, E., Righetto, L., Muffato, F., and Ermondi, C. 1989. "Thermodynamic Study of Sorption of Linear Aliphatic Momhydric Alcohols and Moncarboxylic Acids from $n$-Heptane and $n$-Decane Solution onto $\alpha$-Iron(III) Oxide Immobilized in 
Photosynthetic Membranes." Mater. Chem. and Phys. 21: 155-67. https://doi.org/10.1016/0254-0584(89)90110-7.

[28] Bellobono, I. R., Carrara, A., Barni, B., and Gazzotti, A. 1994. "Laboratory- and Pilot-Plant-Scale Photodegradation of Chloroaliphatics in Aqueous Solution by Photocatalytic Membranes Immobilising Titanium Dioxide." J. Photochem. Photobiol. A Chem. 84: 83-90. https://doi.org/10.1016/1010-6030(94)03844-9.

[29] Bellobono, I. R., and Groppi, F. 2017. "Photocatalytic Membrane Processes, and Respective Modelling, for Removal of Pharmaceutical Residues in Wastewaters. A Case Study with 2-[2,6-(Dichlorophenyl)Amino]Phenyl Acetic Acid as Model Molecule." Curr. Opin. Green $\begin{array}{llll}\text { Sustain. } & \text { Chem. }\end{array}$ https://doi.org/10.1016/j.cogsc.2017.06.007.

[30] Bellobono, I. R., Muffato, F., Selli, E., Righetto, L., and Tacchi, R. 1987. "Transport of Oxygen Facilitated by $\mu$-Peroxo-Bis-[N,N'-Ethylene-Bis-(Salicylideneiminato)Dimethylformamide-Cobalt (III)] Embedded in Liquid Membranes Immobilized by Photografting onto Cellulose." Gas Separ. Purif. 1: 103-6. https://doi.org/103-106.10.1016/0950-4214(87)80018-X.

[31] D’Arienzo, M., Crippa, M., Essawy, A. A., Wahba, L., Scotti, R., Morazzoni, F., Gentile, P., Bellobono, I. R., and Polizzi, S. 2010. "Membrane-Assisted Charge Separation and Photocatalytic Activity in Embedded $\mathrm{TiO}_{2}$ : A Kinetic and Mechanistic Study." J. Phys Chem C 114: 15755-62. https://doi.org/10.1021/jp105854d. 